\title{
BMJ Open How is patient-centred care addressed in women's health? A theoretical rapid review
}

\author{
Anna R Gagliardi, ${ }^{1}$ Sheila Dunn, ${ }^{2}$ Angel Foster, ${ }^{3}$ Sherry L Grace, ${ }^{\bullet 1,4}$ \\ Courtney R Green, ${ }^{5}$ Nazilla Khanlou, ${ }^{5,6}$ Fiona A Miller, ${ }^{7}$ Donna E Stewart, ${ }^{1}$ \\ Simone Vigod, ${ }^{2}$ Frances C Wright ${ }^{8}$
}

To cite: Gagliardi AR, Dunn S, Foster A, et al. How is patientcentred care addressed in women's health? A theoretical rapid review. BMJ Open 2019;9:e026121. doi:10.1136/ bmjopen-2018-026121

- Prepublication history and additional material for this paper are available online. To view these files, please visit the journal online (http://dx.doi org/10.1136/bmjopen-2018026121).

Received 24 August 2018 Revised 31 December 2018 Accepted 8 January 2019

Check for updates

(C) Author(s) (or their employer(s)) 2019. Re-use permitted under CC BY-NC. No commercial re-use. See rights and permissions. Published by BMJ.

For numbered affiliations see end of article.

Correspondence to

Dr Anna R Gagliardi;

anna.gagliardi@uhnresearch.ca

\section{ABSTRACT}

Purpose Efforts are needed to reduce gendered inequities and improve health and well-being for women. Patientcentred care (PCC), an approach that informs and engages patients in their own health, is positively associated with improved care delivery, experiences and outcomes. This study aimed to describe how PCC for women (PCCW) has been conceptualised in research.

Methods We conducted a theoretical rapid review of PCCW in four health conditions. We searched MEDLINE, EMBASE, CINAHL, SCOPUS, Cochrane Library and Joanna Briggs index for English-language articles published from January 2008 to February 2018 inclusive that investigated PCC and involved at least $50 \%$ women aged 18 or older. We analysed findings using a six-domain PCC framework, and reported findings with summary statistics and narrative descriptions.

Results After screening 2872 unique search results, we reviewed 51 full-text articles, and included 14 (five family planning, three preventive care, four depression, one cardiovascular disease and one rehabilitation). Studies varied in how they assessed PCC. None examined all six PCC framework domains; least evaluated domains were addressing emotions, managing uncertainty and enabling self-management. Seven studies that investigated PCC outcomes found a positive association with appropriate health service use, disease remission, health self-efficacy and satisfaction with care. Differing views about PCC between patients and physicians, physician PCC attitudes and geographic affluence influenced PCC. No studies evaluated the influence of patient characteristics or tested interventions to support PCCW.

Conclusion There is a paucity of research that has explored or evaluated PCCW in the conditions of interest. We excluded many studies because they arbitrarily labelled many topics as PCC, or simply concluded that PCC was needed. More research is needed to fully conceptualise and describe PCCW across different characteristics and conditions, and to test interventions that improve PCCW. Policies and incentives may also be needed to stimulate greater awareness and delivery of PCCW.

\section{INTRODUCTION}

Patient-centred care (PCC) refers to engaging patients (and families or care partners) in their own individual healthcare and also to

\section{Strengths and limitations of this study}

- This may be the first synthesis to describe patient-centred care (PCC) specifically for women across multiple clinical areas.

- We used rigorous methods for a theoretical, rapid review that complied with standards for the conduct of electronic search strategies and for reporting of methods and findings.

- We employed an established PCC framework to analyse the included studies, thereby identifying limitations in how PCC has been explored or measured.

- The methodological approach and interpretation of findings were guided by a multidisciplinary research team comprised health services researchers, physicians, experts in women's health and consumer representatives.

- Few studies were included because our search may not have identified all relevant studies and our eligibility criteria may have been overly stringent.

engage patients (or communities) in healthcare service co-design so that all patients benefit from PCC. ${ }^{1}$ At the individual level, PCC improves patient knowledge, relationship with providers, service experience and satisfaction, treatment compliance, appropriate healthcare use, health outcomes and cost-effectiveness of service delivery. ${ }^{2-4}$ However, many patients do not receive or experience PCC. ${ }^{5}$ Improving PCC requires a thorough understanding of what comprises PCC to serve as the basis for measurement, and the design and implementation of strategies to promote and support PCC. Currently, few instruments are available to specifically measure PCC, and they each measure different dimensions of care delivery and the care experience. ${ }^{6}$ Furthermore, some instruments were developed largely by healthcare professionals and may not capture patients' views about what constitutes PCC. ${ }^{7}$ Clearly, more research is needed to better conceptualise, measure and improve PCC for individual patients. 
PCC is not a new concept yet there is currently no standard definition, and the term PCC is used synonymously with other concepts, including quality of care, a much broader concept of which PCC is a component. ${ }^{8}$ PCC frameworks emphasise that it is an approach to care based on patient-provider interaction, ${ }^{9}$ and literature reviews and stakeholder consensus concur. Indeed, several initiatives employed rigorous processes to characterise PCC. A systematic review of the literature for PCC definitions followed by a Delphi survey involving an international panel of stakeholders including patients generated consensus on the most important dimensions of PCC: patient as unique person, patient involvement in care, patient information, patient-clinician communication and patient empowerment. ${ }^{10}{ }^{11}$ A scoping review of 19 studies published from 1994 to 2011 identified 25 unique frameworks or models of PCC..$^{12}$ The frameworks and models differed by number and type of domains, but included one or more elements within common domains pertaining to the patient-provider relationship (sharing information, empathy, empowerment), partnership (sensitivity to needs, relationship-building) and health promotion (collaboration, case management, resource use). McCormack et al established a comprehensive PCC framework based on a systematic review of literature and relevant theories, observing 38 medical encounters between cancer patients and oncologists, interviewing those 38 patients and then reviewing the proposed domains with a 13-member expert panel to refine the framework. ${ }^{13}$ The resulting PCC framework included 31 sub-domains within six interdependent domains: fostering clinician-patient relationships, exchanging information, recognising and responding to patient emotions, managing uncertainty, making decisions and enabling patient self-management.

In 1995, the Fourth World Conference on Women of the United Nations revealed the need to deliver services that are sensitive to the needs and preferences of women, ${ }^{14}$ and in 2009 the WHO report, 'Women and Health', emphasised the need to improve the quality of women's healthcare services. ${ }^{15}$ For example, over-medicalisation of female-specific conditions such as menopause has led to creation and overtreatment of new 'diseases', and confusion and anxiety among women about the best options for maximising their health. ${ }^{16}$ For other conditions common to men and women such as cardiovascular disease, research suggests that there is inequitable access to evidence-based health services; women are less often referred for diagnostic and therapeutic interventions and, once referred, are treated less effectively than men. ${ }^{17}$ Monitoring by the United Nations continues to show that gender-imposed disparities influence women's health; as a result, ensuring healthy lives and promoting wellbeing for women remains one of 17 goals in the 'Gender Equality in the 2030 Agenda for Sustainable Development' issued in 2018. ${ }^{18}$ PCC for women (PCCW) is aimed at improving women's healthcare experiences and associated outcomes. Given lack of consensus on what constitutes PCC, we similarly lack an understanding of PCCW, and how that differs among women with different health conditions or characteristics. The purpose of this study was to review published research on whether and how PCC was conceptualised or measured in research involving women including determinants and outcomes of PCCW. That knowledge could be used in the future as the basis for ongoing research, and for healthcare planning, evaluation and quality improvement.

\section{METHODS}

\section{Approach}

There are many types of research syntheses employing varying methods to address different types of research questions. As part of a larger study of how to support PCCW, our primary goal was to describe how PCCW has been conceptualised; in future research, we will elaborate the PCCW concept by interviewing patients and clinicians. Hence, we chose a theoretical review as the methodological approach. ${ }^{19}$ A theoretical review is characterised by a comprehensive search strategy, inclusion of conceptual and empirical primary sources, explicit study selection, no quality appraisal and content analysis of the included items. It aims to generate insight on key theoretical constructs, either by transforming existing theoretical and empirical evidence into a higher-order conceptual framework, or mapping constructs studied to an existing framework as was done in this study. To quickly describe PCCW so that it could be refined in subsequent components of the larger study, we also adopted a rapid review approach. A rapid review is characterised by restriction to a single language (English), a short time frame (last 10 years, 2008+), exclusion of grey literature, one person performs screening and data abstraction (ARG), quality of included studies is not appraised and authors of included studies are not contacted. ${ }^{2021}$ As there are no reporting of criteria specific to theoretical or rapid reviews, we employed the Preferred Reporting Items for Systematic Reviews and Meta-Analyses criteria to guide reporting of the methods and findings. ${ }^{22}$ Data were publicly available so institutional review board approval was not needed. We did not register a protocol for this review.

\section{Planning}

To become familiar with the literature, we conducted a preliminary search of MEDLINE. The search employed a broad lens to capture all studies of healthcare quality for or among women that may not necessarily having referred to PCC. Using the Medical Subject Headings (MeSH) 'patient-centred care' AND [woman or female], the search generated nearly 31000 results published from 2008 to February 9, 2018 on a diffuse range of topics not necessarily related to PCC, which would have required considerable screening time and effort. Instead, we were interested in a more focused review to assess whether and how others have specifically studied PCC, possibly identifying gaps in knowledge that our future research could 
address. Therefore, we opted for a more targeted strategy, and subsequently searched for only studies in which the focus was explicitly labelled as PCC.

\section{Eligibility criteria}

Knowledge gained from the preliminary search was used to generate eligibility criteria for the planned review based on the PICO (participants, intervention, comparisons, outcomes) framework. The PICO framework is commonly used in systematic reviews to optimise searching and screening. Participants referred to adult women (age 18+) with specific healthcare concerns or conditions in need of improvement. These conditions were chosen based on the proceedings of the Fourth World Conference on Women, ${ }^{14}$ and on recommendations by collaborators of our larger research study (who included health services researchers, clinician investigators and representatives of professional societies, disease-specific foundations, quality improvement and monitoring agencies, patient advocacy groups, patients and consumers) because they are prevalent health concerns for women, or common to both men and women but requiring improved equity or quality of care for women, and represent the full life span: family planning, preventive care, depression and cardiovascular disease or cardiac rehabilitation. Note that, with insight from this, we more comprehensively examined PCCW for other conditions; that work will be published elsewhere. Participants also included physicians or nurses in any setting of care (primary, secondary, tertiary) who cared for women with these conditions. Interventions explicitly referred to PCC, or a synonymous term such as person-centred, women-centred, client-centred, or family-centred care, or approaches or strategies to promote or support PCC. For the purpose of screening, PCG was defined based on constructs common to multiple definitions, ${ }^{8-12}$ and viewed as compassionate, respectful care that addresses patient values and preferences, as well as information and supportive care needs, thus requiring patient-level engagement and patient-provider interaction. To reflect this, we adopted McCormack et al's conceptualisation of PCC in six domains: fostering patient-clinician relationship, exchanging information, recognising and responding to patient emotions, managing uncertainty, making decisions and enabling patient self-management. ${ }^{13}$ As a theoretical review, the primary objective was to describe and compare how PCC was conceptualised and measured across studies and in comparison with the McCormack framework. ${ }^{13}$ Hence, with respect to comparisons, a broad array of study designs were included. Studies were deemed eligible if they explored patient or clinician views about what constitutes PCCW or how to improve PCCW, identified determinants of PCCW including enablers or barriers or evaluated the impact of strategies designed to promote or support PCCW (by comparing patients or clinicians with and without exposure to PCCW strategies, or before or after exposure to strategies or receiving different types of strategies). Outcomes included but were not limited to awareness, understanding, experiences or impacts of PCCW or determinants or factors influencing any of these functions, or the impact of strategies implemented to support or improve PCCW. Regarding publication type, eligible study designs included English-language qualitative (interviews, focus groups, qualitative case studies), quantitative (questionnaires, randomised controlled trials, time series, before/after studies, prospective or retrospective cohort studies, case-control studies) or mixed methods studies. Although systematic reviews were not eligible (to avoid duplication of studies included in reviews and by our search), if deemed relevant, we screened their references to identify additional eligible primary studies.

\section{Searching}

We developed our search strategy in conjunction with a medical librarian and complied with the Peer Review of Electronic Search Strategy reporting guidelines (box 1). ${ }^{23}$ We searched MEDLINE, EMBASE, CINAHL and SCOPUS on 26 February 2018 from 2008 to that date. We also searched the Cochrane Library and the Joanna Briggs Institute Database of Systematic Reviews and Implementation Reports for relevant systematic reviews to screen references. We searched for studies that explicitly used the term 'patient-centred', or an alternative spelling or synonymous option. We supplemented that keyword search with MeSH terms reflecting the concept of PCC to identify studies that employed a synonymous term for PCC that we had not considered and then combined those searches with terms for women.

\section{Screening}

ARG screened titles and abstracts of search results according to the PICO-based eligibility criteria specified above, and generated criteria for ineligible studies prospectively with screening. Studies were not eligible if the participants were family members, care givers or care partners, allied healthcare professionals (ie, pharmacists, dentists) or trainees; or patients or clinicians in long-term care, residential or end-of-life care settings; or where women comprised less than $50 \%$ of participants, or the number of women were not stated. Studies were not eligible if they involved patients in organisational planning, evaluation or improvement or when involvement in co-design was said to have generated a patient-centred service/intervention because patient engagement in service planning or improvement was beyond the scope of this study, which focused on patient engagement in their own individual care; mentioned but did not define or describe what was meant by PCC; or did not study PCC but concluded their research contributes to an understanding of how to deliver or achieve PCC, or shows that PCC is needed. Many studies that arbitrarily referred to PCC in the study of any programme, service, treatment or management of a patient were not eligible. This included studies that focused on the illness experience or clinical treatment preferences or satisfaction with treatment/ 


\section{Box 1 MEDLINE search strategy}

1. Women's health/ (25422)

2. Women/ (14247)

3. Female/ (7835541)

4. 1 or 2 or $3(7839777)$

5. Patient satisfaction/ (71947)

6. Personal satisfaction/ (15404)

7. Patient Preference/ (5969)

8. Patient-Centered Care/ (15651)

9. (patient centered or patient-centered or patient centred or patient-centred).mp. (27001)

10. (person centered or person-centered or person centred or person-centred).mp. (3883)

11. (wom\#n centered or wom\#n-centered or wom\#n centred or wom\#n-centred).mp. (450)

12. Professional-patient relations/ (24731)

13. Health Communication/ (1437)

14. Health Equity/ (367)

15. Health Services Accessibility/ (63814)

16. Patient Participation/ (22042)

17. 5 or 6 or 7 or 8 or 9 or 10 or 11 or 12 or 13 or 14 or 15 or 16 (220827)

18. 4 and 17 (110430)

19. Limit 18 to (English language and $y r=$ '2008 -Current' and 'all adult (19 plus years)") (50343)

20. Limit 19 to (comment or editorial or interview or lectures or letter or news) (493)

21. 19 not 20 (49850)

22. 8 or 9 or 10 or 11 (30272)

23. 4 and 22 (8723)

24. Limit 23 to (English language and $y r=$ '2008 -Current' and 'all adult (19 plus years)") (5055)

25. Limit 24 to (comment or editorial or interview or lectures or letter or news) (26)

26. 24 not 25 (5029)

27. Depression/ (99502)

28. 26 and 27 (161)

29. Cardiac rehabilitation/ (1535)

30. Cardiovascular Diseases/ (128523)

31. 26 and 29 (4)

32. 26 and $30(60)$

33. Family planning services/or reproductive health services/ (25063)

34. 26 and $33(28)$

35. Preventive Health Services/ (12323)

36. Health Promotion/ (65178)

37. Healthy Lifestyle/ (499)

38. 35 or 36 or 37 (76434)

39. 26 and 38 (116)

services or health-related quality of life and not the care experience; explored enablers or barriers of the use of healthcare services only; focused on collaborative or integrated or coordinated or multidisciplinary or interdisciplinary care; patient-centred medical home; motivational interviewing or counselling of patients; concerned interventions delivered by peers or lay persons; patient preferences for clinical outcomes (patient-reported outcomes); and Web-based, computer-based or smartphone-based electronic applications for patients. Articles that singly focused information needs, decision-making, self-management, therapeutic alliance or empathy were also excluded because they examined only one aspect, and not the multiple domains that comprise PCC. ${ }^{13}$ Studies were not eligible if they were protocols, editorials, commentaries, letters, news items, meeting abstracts or proceedings or if they were conceptual or empirical studies published in a language other than English.

\section{Data extraction}

From each study ARG extracted and tabulated data on study characteristics including author, publication year, country, study objective, research design, participants, term used to refer to PCC, definition or description of PCC and findings. If an intervention was employed, ARG also extracted data on content (information/knowledge conveyed), format (mode of delivery, single or multi-faceted), timing (duration, frequency), participants (number, type, setting) and personnel who delivered the intervention according to the Workgroup for Intervention Development and Evaluation Research reporting standards for behavioural interventions. ${ }^{24}$

\section{Data analysis}

We used summary statistics to report the number of studies published per year, and by condition, country, study design and term used for PCC. We compared definitions or descriptions of PCC across studies and conditions. Study quality, while not formally assessed, was evaluated by describing how PCC was conceptualised and measured, We analysed definitions or descriptions of PCC employed in studies with McCormack's six-domain PCC framework. ${ }^{13}$ This means that PCC definitions, descriptions or measures extracted from included studies were mapped to McCormack's PCC domains. To identify gaps or limitations in the way PCCW was studied, we summarised the number of domains addressed in each included study. Instruments used to measure PCC were specified, and we noted if they were validated measures. We described the impact and determinants of PCC narratively, and the number of studies that evaluated interventions designed to promote, support or improve PCC. Team members, comprising health services researchers, physicians of various specialties and experts in women's health, independently reviewed data and the draft manuscript, and provided feedback that shaped the interpretation of results and conclusions.

\section{Patient and public involvement}

This study was informed by a research team that included researchers, collaborators and two consumer representatives. All team members took part in a planning teleconference during which the review objective and eligibility criteria were established.

\section{RESULTS}

\section{Search results}

We identified a total of 2872 unique citations, and excluded 2821 on screening of titles and abstracts. 


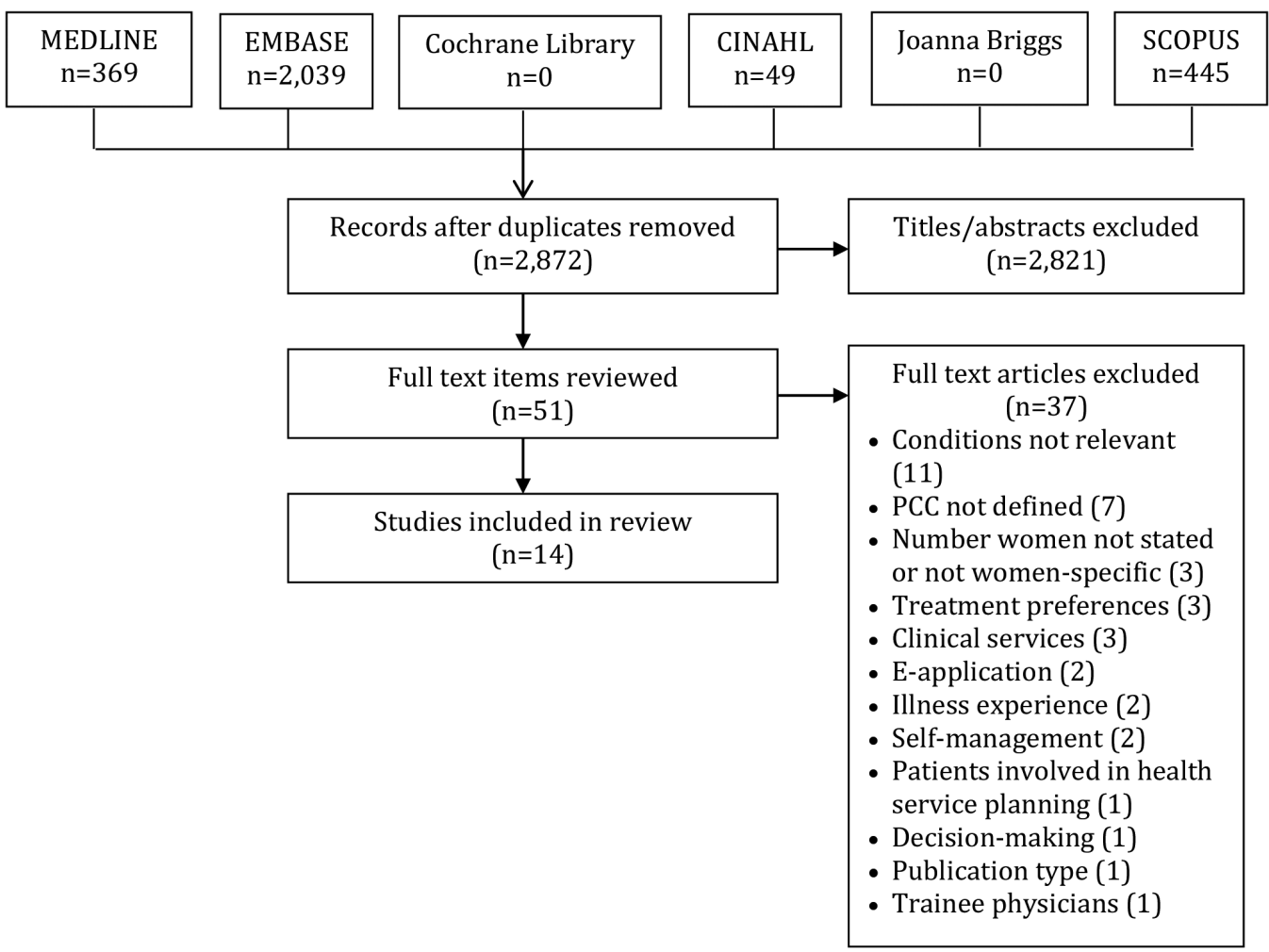

Figure 1 Preferred Reporting Items for Systematic Reviews and Meta-Analyses flow diagram.

Among the remaining 51 full-text articles considered, we excluded 37 because conditions were not relevant $(\mathrm{n}=11)$, PCC was not defined $(\mathrm{n}=7)$, study participants were less than $50 \%$ women or the study was not specific to PCCW $(n=3)$ or the study focused on treatment preferences $(n=3)$, clinical services $(n=3)$, e-applications $(n=2)$, the illness experience $(n=2)$, self-management $(n=2)$, involvement of patients in service co-design rather than their own care $(n=1)$ or decision-making, which is relevant but not a comprehensive assessment of PCC $(n=1)$. We excluded two additional studies due to publication type $(n=1)$ and because participants were trainee physicians $(n=1)$. Ultimately, we included 14 studies for review (figure 1). Data extracted from eligible studies are available in online supplementary file $1.25-38$

\section{Study characteristics}

Studies were published from 2008 to 2017. Most employed the term 'patient-centred care' $(\mathrm{n}=13)$; one study referred to 'woman-centred care'. Most studies were conducted in the United States $(n=10)$ followed by one each in Australia, China, Iran and Scotland. By condition, studies included one on cardiovascular disease, three on preventive care, five on family planning and five on depression. With respect to study design, the largest number of studies were statistical analyses of survey data to examine the association of PCC with receipt of treatment or outcomes $(n=6)$. Other studies involved qualitative interviews with women to describe PCCW $(n=3)$ or qualitative observation of patients and clinicians to assess if PCCW occurred during consultations $(n=3)$. Two studies were concept analyses to describe an approach for delivering PCCW. Seven $(50.0 \%)$ studies focused solely on women: one on preventive care, five on family planning and one on depression; the remaining seven studies were included because they involved at least $50 \%$ women: one on cardiovascular disease, two on preventive care, and four on depression.

\section{PCCW definition}

Table 1 summarises the definition or domains of PCC employed or measured, or the definition or domains of PCCW generated by each study. No study addressed all the six PCG domains, although four studies addressed five domains and six studies addressed four domains. The domains most frequently addressed by the 14 studies were exchanging information $(n=13)$, making decisions $(\mathrm{n}=12)$ and fostering the relationship $(\mathrm{n}=11)$. Domains addressed less frequently by the 14 studies were addressing emotions $(n=7)$, managing uncertainty $(\mathrm{n}=7)$ and enabling self-management $(\mathrm{n}=5)$. One study that explored factors influencing decisions about routine Papanicolaou testing or mammography also found that women desired access to a female physician and a womanonly environment. There was no difference in number of PCC domains addressed across conditions; the mean and median number of PCC domains were 3.3 and 3.0, respectively, for each of preventive care, family planning and depression. There did not appear to be patterns of PCC domains addressed by condition.

\section{PCCW measurement}

Seven (50\%) studies employed the existing validated instruments to measure PCC. They included 


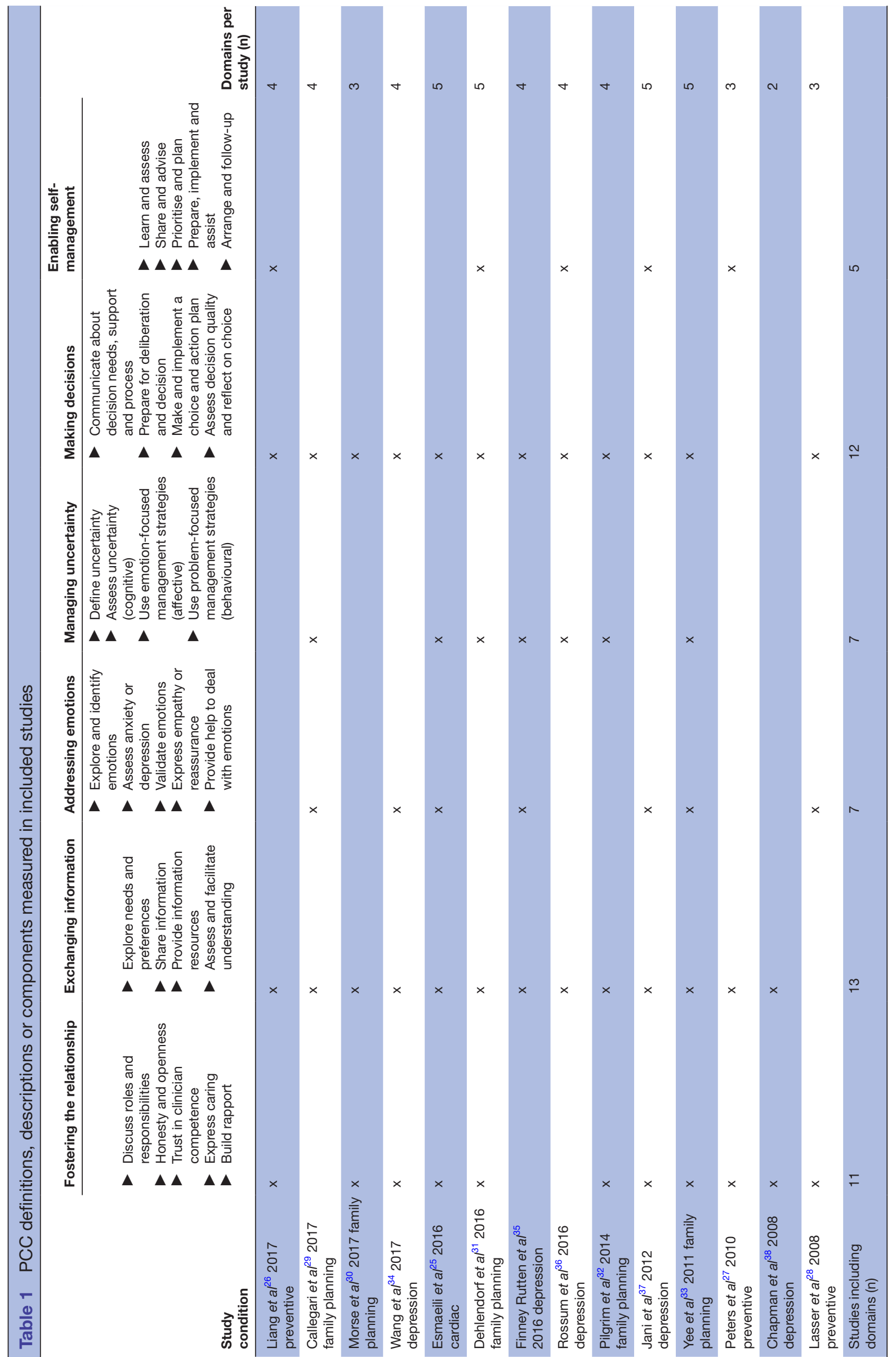


the Interpersonal Quality in Family Planning Scale, ${ }^{31}$ Client-Clinician Centeredness Scale, ${ }^{32}$ Patient-Practitioner Orientation Scale, ${ }^{34}$ Patient Assessment of Chronic Illness Care Survey, ${ }^{36}$ Consultation and Relational Empathy Questionnaire ${ }^{37}$ and Measure of Patient-Centred Communication. ${ }^{37} 38$

\section{Impact of PCCW}

Seven (50\%) studies examined PCCW outcomes. Two qualitative studies explored aspects of PCC that influenced receipt of preventive services including routine Papanicolaou testing or mammography, ${ }^{27}$ and influenza vaccine or colorectal cancer screening, ${ }^{28}$ and one survey study found that PCC increased receipt of preventive services monitoring of blood pressure or cholesterol, routine check-up, blood stool test, breast exam, mammography, Papanicolaou testing as well as exercise and diet education. ${ }^{26}$ Two survey studies of family planning found that PCC improved sustained use of chosen contraceptive method 6 months later, ${ }^{31}$ and satisfaction with care in family planning programmes. ${ }^{32}$ Among patients with depression, studies showed that PCC was positively associated with health self-efficacy for dealing with feelings of uncertainty about health or healthcare, ${ }^{35}$ and remission of depression at 6 months and rating of care quality. ${ }^{36}$

\section{PCCW determinants}

Three studies, all based on depression care, examined challenges or barriers of PCC. A survey study found that patients and physicians differed in their preferences for patient-centred communication. ${ }^{34}$ A study involving observation of consultations showed that PCC was less likely in less affluent areas compared with those more affluent. ${ }^{37}$ That study also examined physician behaviour; physicians in deprived areas looked at patients fewer times, and used fewer head nods and fewer positive facial expressions. Another study involving observation of consultations found that physician attributes influenced PCC: higher dutifulness was positively associated with treating patients as whole persons and finding common ground while those exhibiting anxiety or vulnerability scored lower for finding common ground. ${ }^{38}$ No studies examined whether or how women's characteristics influenced preferences for or receipt of PCC.

\section{Strategies to support PCCW}

None of the 14 included studies developed, implemented or evaluated the impact of an intervention to promote or support PCCW.

\section{DISCUSSION}

This theoretical rapid review identified a paucity of research on PCCW across four conditions. Moreover, none of the studies addressed all the six domains of the comprehensive McCormack et al PCC framework, ${ }^{13}$ with half of the studies or fewer evaluating the domains of addressing emotions, managing uncertainty and enabling self-management. Each study defined, described or measured PCC differently, and half of the studies employed an existing validated instrument (scale or questionnaire) to assess PCC. Three studies examined barriers to PCC, which were differences between patients and physicians about the importance of PCC domains, physician personality characteristics and receiving care in less affluent areas. No studies examined whether or how women's characteristics influenced preferences for or receipt of PCC, though one study found that geographical affluence influenced PCC. Of the seven (50\%) studies that examined the impact of PCC, all found that PCC was positively associated with uptake of preventive care tests or education, health self-efficacy, satisfaction with care, contraception use and remission of depression. No studies examined interventions to promote or support PCCW. Hence, although study quality was not directly assessed, by describing how PCC was conceptualised and measured, we identified numerous limitations of research on PCCW. Given the paucity of research on PCCW, it was not possible to generate theoretical or conceptual insight on whether or how PCC elements, determinants or interventions influence outcomes.

The 1995 United Nations Fourth World Conference on Women, considered a springboard to gender equality by setting a 12-point agenda for the advancement of women, was adopted by 189 countries. ${ }^{14}$ One of the 12 points was women and health, which referred to improving quality of care, strengthening preventive programmes and addressing gender-sensitive issues such as family planning. Hence, it is surprising that little research on the conditions we examined specifically studied PCCW. A few factors might contribute to the paucity of research on PCCW. One reason may be lack of clarity and agreement on what constitutes PCC. ${ }^{8}$ Notably, we excluded a large number of studies because they arbitrarily used PCC to refer to a wide variety of healthcare issues, or failed to define PCC or employ or generate a comprehensive PCC framework. Another reason may be a lack of policy or system guidance and incentives for PCCW. For example, Wiig et al found that health policy in 10 European countries did not specify mechanisms to improve healthcare quality. ${ }^{39}$ Gauld et al found that primary care policies in seven countries only recently identified quality and safety as important platforms. ${ }^{40}$ The more recent 'Gender Equality in the 2030 Agenda for Sustainable Development', released in 2018, confirms the need for efforts to improve health and healthcare for women. ${ }^{18}$ To achieve this, among other action items, the report recommends integrated policies and associated incentives to achieve goals. Future research should examine whether and how legislation and policies recognise and promote PCCW, and how those laws and policies are interpreted and implemented. This may reveal the approaches and interventions needed to create greater awareness and delivery of PCCW.

Another key finding was that each study defined and measured PCC differently, and none described or measured it as comprehensively as the McCormack framework. ${ }^{13}$ Given that few studies were eligible, it is unclear if the observed 
variations in conceptualising or operationalising PCC mean that PCCW differs for different conditions. Research by others that explored the perspectives of men and women with different conditions appear to also have generated different domains or dimensions of PCC. ${ }^{41-43}$ Moreover, patients' PCC needs may vary depending on whether the aim is to understand their condition, decide on treatment or plan self-management, ${ }^{44}$ and may also vary along their illness trajectory or according to demographic or cultural characteristics. ${ }^{45}$ Due to the paucity of eligible research, it was not possible to generate theoretical or conceptual insight on PCCW. Future research could employ similar methods for reviewing literature on PCCW for other conditions, and along with our ongoing research involving interviews with women who vary by condition and characteristics, may generate further insight and advance our understanding of how to optimise PCCW. Other researchers have noted that available instruments purported to evaluate PCC each measure different dimensions of care delivery and the care experience, and called for more instruments to be developed. ${ }^{5}$ The findings of our study suggest that, first, more research is needed to fully define and describe PCCW to understand commonalities and where important condition-specific or characteristic-specific differences lie.

This review and previous research found that PCC is associated with improved care delivery and outcomes. ${ }^{2-4}$ However, few studies specifically examined facilitators or barriers of PCC, and no studies evaluated interventions to promote or support PCCW. A Cochrane systematic review by Baker et al found that interventions that had been selected and tailored to address identified barriers of guideline-adherent clinical care were more likely to improve professional practice compared with either no intervention or simple dissemination of guidelines. ${ }^{46}$ Therefore, in addition to research already suggested, more study is needed of the determinants of PCCW, as this knowledge is needed to select and tailor interventions that would improve PCCW and associated outcomes.

This review features strengths and limitations. We employed a review approach most suitable to our research objective, and searched the most relevant databases of medical literature with a search strategy that complied with standards, ${ }^{23}$ and we compared PCCW across four conditions, two specific to women and two common to men and women. A few issues may limit the interpretation and use of these findings. Given the rapid review approach involving a single screener and no review of grey literature, we may not have identified all relevant studies. While our search strategy was comprehensive, it may have omitted potentially relevant terms. Our exclusion criteria may have been overly stringent and eliminated potentially relevant studies that may have examined topics relevant to PCC; however, our intent was to examine whether PCC as a multi-domain concept had been thoroughly evaluated to inform future research. While perhaps not ideal, to achieve even a small volume of eligible studies, we included studies that involved both men and women provided that results described differences between men and women. Only half of the included studies involved women-only, which emphasises the paucity of research on PCCW and represents an important finding. Due to the small number of included studies, and with only half of included studies solely focused on women, future research is necessary to establish a more definitive PCCW framework for women with different characteristics or conditions. Still, this may be the first study to examine whether and how PCCW has been investigated, and it raises a number of implications and issues that warrant ongoing research.

\section{CONCLUSION}

International policy and advocacy efforts have emphasised the need to improve the quality and experience of care for women with different healthcare issues across the life span. PCC, an approach that informs and engages patients in their own healthcare that is positively associated with improved healthcare experiences and outcomes, is also an international priority. Yet this review identified a few studies that explored or evaluated PCCW concerning family planning, preventive healthcare services, depression and cardiovascular disease or cardiac rehabilitation. Studies varied in how they assessed PCC and none fully conceptualised PCC according to an existing comprehensive PCC framework. Few studies identified facilitators or barriers of PCC, and no studies evaluated interventions to promote or support PCCW. Notably, many studies were excluded because they referred to a wide array of arbitrary topics as PCC or concluded that PCC was needed without having defined PCC. More research is needed to fully conceptualise and describe PCCW across different characteristics and conditions relevant to women, examine whether and how legislation and policies recognise and promote PCCW and explore barriers and facilitators of PCCW. Policies, associated incentives and tailored interventions may also be needed to stimulate awareness and delivery of PCCW.

\section{Author affiliations}

${ }^{1}$ Toronto General Hospital Research Institute, University Health Network, Toronto, Ontario, Canada

${ }^{2}$ Women's College Research Institute, Women's College Hospital, Toronto, Ontario, Canada

${ }^{3}$ Faculty of Health Sciences, University of Ottawa, Ottawa, Ontario, Canada ${ }^{4}$ School of Kinesiology and Health Science, York University, Toronto, Ontario, Canada ${ }^{5}$ Society of Obstetricians and Gynecologists of Canada, Ottawa, Ontario, Canada ${ }^{6}$ Faculty of Health/School of Nursing, York University, Toronto, Ontario, Canada ${ }^{7}$ Institute of Health Policy, Management and Evaluation, University of Toronto, Toronto, Ontario, Canada

${ }^{8}$ Louise Temerty Breast Cancer Centre, Sunnybrook Health Sciences Centre, Toronto, Ontario, Canada

Contributors ARG conceptualised the study, collected and analysed data, drafted the article and finalised the article by integrating feedback from co-authors. SD, AF, SLG, CRG, NK, FAM, DES, SV and FCW assisted with conceptualising the study and planning elements of study design. SD, AF, SLG, CRG, NK, FAM, DES, SV and FCW assisted in reviewing and interpreting data, critically appraised the draft article for content and for accuracy and integrity, and reviewed and approved the final version.

Funding This study was funded by an annual grant from the Government of Ontario. This work was supported by the Ontario Ministry of Health and Long-term Care grant number 251.

Disclaimer The opinions, results and conclusions in this paper are those of the authors and are independent from the funding source. 
Competing interests None declared.

Patient consent for publication Not required.

Ethics approval Data were publicly available so institutional review board approval was not needed.

Provenance and peer review Not commissioned; externally peer reviewed.

Data sharing statement № additional data are available.

Open access This is an open access article distributed in accordance with the Creative Commons Attribution Non Commercial (CC BY-NC 4.0) license, which permits others to distribute, remix, adapt, build upon this work non-commercially, and license their derivative works on different terms, provided the original work is properly cited, appropriate credit is given, any changes made indicated, and the use is non-commercial. See: http://creativecommons.org/licenses/by-nc/4.0/.

\section{REFERENCES}

1. Carman KL, Dardess P, Maurer M, et al. Patient and family engagement: a framework for understanding the elements and developing interventions and policies. Health Aff 2013;32:223-31.

2. Rathert C, Wyrwich MD, Boren SA. Patient-centered care and outcomes: a systematic review of the literature. Med Care Res Rev 2013;70:351-79.

3. Doyle C, Lennox L, Bell D. A systematic review of evidence on the links between patient experience and clinical safety and effectiveness. BMJ Open 2013;3:e001570.

4. Stewart M, Ryan BL, Bodea C. Is patient-centred care associated with lower diagnostic costs? Healthc Policy 2011;6:27-31.

5. Fowler FJ, Gerstein BS, Barry MJ. How patient centered are medical decisions?: results of a national survey. JAMA Intern Med 2013;173:1215-21.

6. Hudon C, Fortin M, Haggerty JL, et al. Measuring patients' perceptions of patient-centered care: a systematic review of tools for family medicine. Ann Fam Med 2011;9:155-64.

7. Ogden K, Barr J, Greenfield D. Determining requirements for patientcentred care: a participatory concept mapping study. BMC Health Serv Res 2017;17:780.

8. Lusk JM, Fater K. A concept analysis of patient-centered care. Nurs Forum 2013;48:89-98.

9. Epstein RM, Street RL. The values and value of patient-centered care. Ann Fam Med 2011;9:100-3.

10. Scholl I, Zill JM, Härter M, et al. An integrative model of patientcenteredness - a systematic review and concept analysis. PLoS One 2014;9:e107828.

11. Zill JM, Scholl I, Härter M, et al. Which dimensions of patientcenteredness matter? - Results of a web-based expert delphi survey. PLoS One 2015;10:e0141978.

12. Constand MK, MacDermid JC, Dal Bello-Haas V, et al. Scoping review of patient-centered care approaches in healthcare. BMC Health Serv Res 2014;14:271.

13. McCormack LA, Treiman K, Rupert D, et al. Measuring patientcentered communication in cancer care: a literature review and the development of a systematic approach. Soc Sci Med 2011;72:1085-95.

14. World Health Organization. Fourth World Conference on Women. Geneva: WHO Press, 1995

15. World Health Organization. Women and Health. Geneva: WHO Press, 2009.

16. Sievert LL, Saliba M, Reher D, et al. The medical management of menopause: a four-country comparison care in urban areas. Maturitas 2008;59:7-21.

17. Bennett AL, Lavie CJ, Grace SL. Cardiac rehabilitation following acute coronary syndrome in Women. Curr Treat Options Cardiovasc Med 2017; 19:57.

18. Women UN. Turning promises into action. Gender equality in the 2030 agenda for sustainable development. United Nations 2018

19. Paré G, Trudel M-C, Jaana M, et al. Synthesizing information systems knowledge: a typology of literature reviews. Inf Manage 2015:52:183-99.

20. Tricco AC, Antony J, Zarin W, et al. A scoping review of rapid review methods. BMC Med 2015;13:224

21. Reynen $\mathrm{E}$, Robson $\mathrm{R}$, Ivory $\mathrm{J}$, et al. A retrospective comparison of systematic reviews with same-topic rapid reviews. $J$ Clin Epidemiol 2018;96:23-34.

22. Moher D, Liberati A, Tetzlaff J, et al. Preferred reporting items for systematic reviews and meta-analyses: the PRISMA statement. PLOS Med 2009;6:e1000097.
23. McGowan J, Sampson M, Salzwedel DM, et al. PRESS Peer review of electronic search strategies: 2015 guideline statement. J Clin Epidemiol 2016;75:40-6.

24. Albrecht L, Archibald M, Arseneau D, et al. Development of a checklist to assess the quality of reporting of knowledge translation interventions using the Workgroup for Intervention Development and Evaluation Research (WIDER) recommendations. Implement Sci 2013;8:8.

25. Esmaeili M, Cheraghi MA, Salsali M. Cardiac patients' perception of patient-centred care: a qualitative study. Nurs Crit Care 2016;21:97-104.

26. Liang $\mathrm{H}$, Zhu J, Kong $\mathrm{X}$, et al. The patient-centered care and receipt of preventive services among older adults with chronic diseases: a nationwide cross-sectional study. Inquiry 2017:54:1-11.

27. Peters K. Reasons why women choose a medical practice or a women's health centre for routine health screening: worker and client perspectives. J Clin Nurs 2010;19:2557-64.

28. Lasser KE, Kelly B, Maier J, et al. Discussions about preventive services: a qualitative study. BMC Fam Pract 2008;9:49.

29. Callegari LS, Aiken AR, Dehlendorf $C$, et al. Addressing potential pitfalls of reproductive life planning with patient-centered counseling. Am J Obstet Gynecol 2017;216:129-34.

30. Morse JE, Ramesh S, Jackson A. Reassessing unintended pregnancy. Toward a patient-centered approach to family planning. Obstet Gynecol Clin N Am 2017;44:27-40.

31. Dehlendorf C, Henderson JT, Vittinghoff E, et al. Association of the quality of interpersonal care during family planning counseling with contraceptive use. Am J Ob Gyn 2016;215:78.e1-9.

32. Pilgrim NA, Cardona KM, Pinder E, et al. Clients' perceptions of service quality and satisfaction at their initial Title $X$ family planning visit. Health Commun 2014;29:505-15.

33. Yee L, Simon M. Urban minority women's perceptions of and preferences for postpartum contraceptive counseling. J Midwifery Womens Health 2011;56:54-60.

34. Wang J, Zou R, Fu H, et al. Measuring the preference towards patient-centred communication with the Chinese-revised patientpractitioner orientation Scale: a cross-sectional study among physicians and patients in clinical settings in Shanghai, China. BMJ Open 2017;7:e016902.

35. Finney Rutten LJ, Hesse BW, St Sauver JL, et al. Health selfefficacy among populations with multiple chronic conditions: the value of patient-centered communication. Adv Ther 2016;33:1440-51.

36. Rossom RC, Solberg LI, Vazquez-Benitez G, et al. The effects of patient-centered depression care on patient satisfaction and depression remission. Fam Pract 2016;33:649-55.

37. Jani B, Bikker AP, Higgins M, et al. Patient centredness and the outcome of primary care consultations with patients with depression in areas of high and low socioeconomic deprivation. Br J Gen Pract 2012;62:e576-81.

38. Chapman BP, Duberstein PR, Epstein RM, et al. Patient-centered communication during primary care visits for depressive symptoms: what is the role of physician personality? Med Care 2008;46:806-12.

39. Wiig S, Aase K, von Plessen C, et al. Talking about quality: exploring how 'quality' is conceptualized in European hospitals and healthcare systems. BMC Health Serv Res 2014:14:478.

40. Gauld R, Burgers J, Dobrow M, et al. Healthcare system performance improvement. J Health Organ Manag 2014;28:2-20.

41. van den Berg MMJ, Dancet EAF, Erlikh T, et al. Patient-centered early pregnancy care: a systematic review of quantitative and qualitative studies on the perspectives of women and their partners. Hum Reprod Update 2018;24:106-18.

42. Hudon C, Fortin M, Haggerty J, et al. Patient-centered care in chronic disease management: a thematic analysis of the literature in family medicine. Patient Educ Couns 2012;88:170-6.

43. Segan JD, Briggs AM, Chou L, et al. Patient-perceived health service needs in inflammatory arthritis: A systematic scoping review. Sem Arth Rheum 2018;47.

44. Lo AC, Olson R, Feldman-Stewart D, et al. A patient-centered approach to evaluate the information needs of women with Ductal Carcinoma In Situ. Am J Clin Oncol 2017;40:574-81.

45. Shim EJ, Park JE, Yi M, et al. Tailoring communications to the evolving needs of patients throughout the cancer care trajectory: a qualitative exploration with breast cancer patients. BMC Womens Health 2016;16:65.

46. Baker R, Camosso-Stefinovic J, Gillies C, et al. Tailored interventions to address determinants of practice. Cochrane Database Syst Rev 2015;4:CD005470. 